\title{
FIXED-POINT SETS OF TRANSFORMATION GROUPS ON INFINITE-PRODUCT SPACES ${ }^{1}$
}

\author{
JAMES E. WEST
}

If $G$ is a topological group, $X$ is a topological space, $\alpha$ is a transformation group action of $G$ on $X$, and there is a subset $Y$ of $X$ such that for each element $g$ in $G$ which is not the identity,

$$
Y=\{x \in X \mid \alpha(g, x)=x\},
$$

then $\alpha$ is said to be a semifree action of $G$ on $X$ with fixed-point set $Y$. If $Y$ is empty, then $\alpha$ is said to be free. The problem addressed in this paper is to find sufficient conditions that each closed subset of $X$ be the fixed-point set of a semifree action of $G$. The main result proven here is Theorem 3.2: If $X$ is a separable, infinite-dimensional Frèchet space and $G$ is a topological group for which there is an effective action on $X$ as a transformation group, then each closed subset of $X$ is the fixedpoint set of a semifree action of $G$ on $X$. This generalizes the theorem of V. L. Klee, Jr. [8] that each compactum of a separable, infinitedimensional Hilbert space is the fixed-point set of periodic homeomorphisms of the space onto itself of all periods greater than one, and is complementary to A. Beck's theorem [4] that if $X$ is a metric space on which there is an action of the real line without degenerate orbits, then each closed set of $X$ is the union of the degenerate orbits of some action of the real line on $X$ (in the sense that whereas Beck's theorem removes restrictions on the space $X$, the theorem of this paper removes restrictions on the group $G$ ).

1. Terminology and notational conventions. A transformation group is taken to be a triple $(G, X, \alpha)$, where $G$ is a topological group, $X$ is a topological space, and $\alpha$ is a continuous function from $G \times X$ onto $X$ such that (a) if $e$ is the identity, then $\alpha(e, x)=x$ for each $x$ in $X$, and (b) if $g$ and $h$ are in $G$, then for each $x$ in $X, \alpha(g h, x)=\alpha(g, \alpha(h, x))$. No separation requirements are made on $G$ and $X$. The function is called the action of $G$ on $X$, and is said to be effective if whenever $\alpha(g, x)=x$ for each $x$ in $X$, then $g=e$.

The group of all homeomorphisms of a space $X$ onto itself is denoted by $G(X)$. If $\alpha$ is a transformation group action of $G$ on $X$, then

Received by the editors May 7, 1968.

1 This material is part of the author's Ph.D. dissertation, prepared under the guidance of Professor R. D. Anderson at the Louisiana State University in 1966 and 1967. 
$\alpha$ defines a homomorphism of $G$ into $G(X)$ which is also denoted by $\alpha$, as there is no possibility of confusion.

The terms "normal topological space" and "perfectly normal topological space" are not taken to imply the Hausdorff Separation Property.

The set of positive integers is denoted by $N$, and the interval $[0,1]$, by $I$. If $X$ is a space, then the countably infinite Cartesian product of $X$ with itself is denoted by $\prod_{i \in N} X_{i}$ and by $X^{\infty}$, and the projection of $X^{\infty}$ onto $X_{i}$ is written $\pi_{i}$. The projection of $X^{\infty}$ onto $\prod_{i \leqq n} X_{i}\left(=X^{n}\right)$ is written $\tau_{n}$.

If $Y$ is a subset of the space $X$, then $\mathrm{cl}(Y)$ denotes the closure of $Y$ in $X$.

Finally, if $g$ and $h$ are two elements of $G(X)$ for some space $X$, an isotopy between $g$ and $h$ is a set $\left\{f_{t}\right\}_{t \in I}$ of elements of $G(X)$ such that $f_{0}=g, f_{1}=h$, and the function $F: X \times I \rightarrow X$ defined by $F(x, t)=f_{t}(x)$ is continuous. Usually, the isotopy is required to be "invertible" in the sense that $\left\{f_{t}^{-1}\right\}_{t \in I}$ is an isotopy between $f_{0}^{-1}$ and $f_{1}^{-1}$.

2. A theorem for infinite-product spaces. For a space $X$, let $T$ denote the element of $G\left(X^{\infty}\right)$ which exchanges the $(2 n-1)$ st and $2 n$th coordinates for each $n$ in $N$. The theorem proven in this section requires that $X$ be a space for which this homeomorphism is invertibly isotopic to the identity. For convenience, this property is called the Reflective Isotopy Property. It actually implies that every transformation group action on $X^{\infty}$ is "isotopic" to the trivial action in the sense that there is a continuous function $H$ from $G \times X^{\infty} \times I$ to $X^{\infty}$ such that for each $t$ in $I, H / G \times X^{\infty} \times\{t\}$ defines a transformation group action of $G$ on $X^{\infty}$ which is the original action if $t=1$ and is the projection onto the second factor if $t=0$. A proof of this statement may be obtained by adapting Raymond Wong's proof [10] that, for separable, metric spaces, the condition that the element of $G\left(X^{\infty}\right)$ which exchanges the first and second coordinates be isotopic to the identity is equivalent to the condition that each element of $G\left(X^{\infty}\right)$ be isotopic to the identity.

Theorem 2.1. If $G$ is a topological group and $X$ is a topological space which has the Reflective Isotopy Property and for which $X^{n}$ is perfectly normal for each $n$ in $N$, then there is a free action of $G$ on $X^{\infty}$ if and only if each closed set of $X^{\infty}$ is the fixed-point set of a semifree action of $G$ on $X^{\infty}$.

Proof of Theorem 2.1. All that is necessary is to prove that if there is a free action of $G$ on $X^{\infty}$, then each closed set of $X^{\infty}$ is the 
fixed-point set of a semifree action of $G$ on $X^{\infty}$, so let $\alpha$ be any free action of $G$ on $X^{\infty}$ and $Y$ be any closed subset of $X^{\infty}$ not equal to $X^{\infty}$. The procedure is first to construct a sequence of closed "annular" subsets $K_{i}$ of $X^{\infty}-Y$ ringing $Y$ which converge to the boundary of $Y$ and second to construct free actions of $G$ on the $K_{i}$. These actions will converge to the trivial action as $K_{i}$ approaches the boundary of $Y$ and will agree on the interfaces of the $K_{i}$. When pieced together with the trivial action on $Y$, these actions will yield the desired action of $G$ on $X^{\infty}$.

Step 1. The construction of the $K_{i}$. Let $\left\{U_{i}\right\}_{i \in N}$ be a collection of open sets of $X^{\infty}$ satisfying the following four conditions:

(1) $U_{1}=X^{\infty}$;

(2) $Y=\bigcap_{i \in N} U_{i}$;

(3) $U_{i}=\tau_{i}^{-1}\left(\tau_{i}\left(U_{i}\right)\right)$, for each $i$ in $N$;

(4) $\operatorname{cl}\left(U_{i+1}\right) \subset U_{i}$, for each $i$ in $N$.

Such a collection may be constructed inductively using the hypothesis that each $X^{i}$ is perfectly normal. For each $i$ in $N$, let $\left\{V_{i j}\right\}_{j \in N}$ be a collection of open sets in $X^{i}$ such that $\operatorname{cl}\left(\tau_{i}(Y)\right)=\bigcap_{j \in N} V_{i j}$, and the $\tilde{V}_{i j}=\tau_{i}^{-1}\left(V_{i j}\right)$. Set $U_{1}=X^{\infty}$, and suppose that $n$ is an integer greater than one and $\left\{U_{i}\right\}_{i=1}^{n}$ is a collection of open sets in $X^{\infty}$ satisfying (1), (3), and (4) whenever applicable together with the condition that $U_{i} \subset \tilde{V}_{k l}$ whenever $i \geqq k$ and $l$. By choosing $W$ to be an open set in $X^{n+1}$ such that

$$
\operatorname{cl}\left(\tau_{n+1}(Y)\right) \subset W \subset \operatorname{cl}(W) \subset \tau_{n+1}\left(\left(\bigcap_{1 \leqq i \leqq n} U_{i}\right) \cap\left(\bigcap_{k, l \leqq n+1} V_{k l}\right)\right)
$$

and by setting $U_{n+1}=\tau_{n+1}^{-1}(W)$, one can construct a collection $\left\{U_{i}\right\}_{i=1}^{n+1}$ satisfying (1), (3), and (4) whenever applicable and the condition on the $V_{i j}$ which contains the original collection. By induction, there is a collection $\left\{U_{i}\right\}_{i \in N}$ satisfying (1), (3), and (4) together with the condition that $Y \subset U_{i} \subset \bigcap_{k, l \leqq i} \widetilde{V}_{k l}$. Since $Y=\bigcap_{k, l \in N} \widetilde{V}_{k l}, Y=\bigcap_{i \in N} U_{i}$, and condition (2) is satisfied. Now set $K_{i}=\operatorname{cl}\left(U_{i}-U_{i+1}\right)$ for each $i$ in $N$.

Step 2. The definition of the action of $G$ on $X^{\infty}$. By Urysohn's Lemma, select, for each $i$ in $N$, a continuous function $\bar{f}_{i}: \tau_{i+1}\left(K_{i}\right) \rightarrow I$ such that $\operatorname{cl}\left(\tau_{i+1}\left(U_{i}\right)\right)-\tau_{i+1}\left(U_{i}\right) \subset \bar{f}_{i}^{-1}(0)$ and $\mathrm{cl}\left(\tau_{i+1}\left(U_{i+1}\right)\right)-\tau_{i+1}\left(U_{i+1}\right) \subset \bar{f}_{i}^{-1}(1)$. Let $f_{i}=\bar{f}_{i} \tau_{i+1}$.

Let $\left\{h_{t}\right\}_{t \in I}$ be an invertible isotopy between the identity of $G\left(X^{\infty}\right)$ and $T$. Also, let $\sigma$ be the "shift" homomorphism of $G\left(X^{\infty}\right)$ into itself defined by the formula $\sigma(h)\left(x_{1}, x_{2}, \cdots\right)=\left(x_{1}, \pi_{1} h\left(x_{2}, x_{3}, \cdots\right)\right.$, $\left.\pi_{2} h\left(x_{2}, x_{3}, \cdots\right), \pi_{3} h(), \cdots\right)$ for each $h$ in $G\left(X^{\infty}\right)$ and $\left(x_{1}, x_{2}, \cdots\right)$ 
in $X^{\infty}$; let $\omega$ be the homomorphism of $G\left(X^{\infty}\right)$ into itself defined by the formula

$$
\begin{aligned}
& \omega(h)\left(x_{1}, x_{2}, \cdots\right) \\
& \quad=\left(\pi_{1} h\left(x_{1}, x_{3}, x_{5}, \cdots\right), x_{2}, \pi_{2} h\left(x_{1}, x_{3}, x_{5}, \cdots\right), x_{4}, \cdots\right) .
\end{aligned}
$$

For each $i$ in $N$, let $\rho_{i}$ be the element of $G\left(K_{i}\right)$ defined by $\rho_{i}(x)$ $=\sigma^{i+1}\left(h_{f_{i}(x)}\right)(x)$ for each $x$ in $K_{i}$. Now define $\beta: G \times X^{\infty} \rightarrow X^{\infty}$ by

$$
\begin{aligned}
\beta(g, x) & =\rho_{i}^{-1} \sigma^{i+1}(\omega(\alpha(g))) \rho_{i}(x), \quad \text { if } x \in K_{i} \\
& =x, \quad \text { if } x \in Y
\end{aligned}
$$

for each $(g, x)$ in $G \times X^{\infty}$.

Step 3. The verification that $\beta$ is a semifree action of $G$ on $X$ with fixedpoint set $Y$.

(a) $\beta$ is well defined, because for each $i$ in $N$ and $h$ in $G\left(X^{\infty}\right)$, the function $\sigma^{i+1}(h) \mid K_{i} \in G\left(K_{i}\right)$, as it does not alter the first $i+1$ coordinates of any point. This also demonstrates that $f_{i} \sigma^{i+1}(h)=f_{i}$, which shows that the function defined on $K_{i}$ by $x \rightarrow \sigma^{i+1}\left(h_{f_{i}(x)}^{-1}\right)(x)$ is actually $\rho_{i}^{-1}$. Because $\left\{h_{t}\right\}_{t \in I}$ is an invertible isotopy, $\rho_{i}^{-1}$ is continuous, and thus $\rho_{i} \in G\left(K_{i}\right)$ as claimed earlier. Similarly, $\rho_{i}^{-1} \sigma^{i+1}(\omega(\alpha(g))) \rho_{i}$ is in $G\left(K_{i}\right)$ for each $g$ in $G$ and each $i$ in $N$. Now, if $g \in G$ and $x \in K_{i} \cap K_{i+1}$, then $f_{i}(x)=1$, so $\rho_{i}(x)=\sigma^{i+1}(T)(x)=\left(x_{1}, x_{2}, \cdots, x_{i+1}, x_{i+3}, x_{i+2}\right.$, $\left.x_{i+5}, x_{i+4}, \cdots\right)$. Therefore,

$$
\begin{aligned}
& \rho_{i}^{-1} \sigma^{i+1}(\omega(\alpha(g))) \rho_{i}(x) \\
&=\rho_{i}^{-1} \sigma^{i+1}(\omega(\alpha(g)))\left(x_{1}, \cdots, x_{i+1}, x_{i+3}, x_{i+2}, \cdots\right) \\
&=\rho_{i}^{-1}\left(x_{1}, \cdots, x_{i+1}, \pi_{1} \alpha(g)\left(x_{i+3}, x_{i+5}, \cdots\right), x_{i+2}, \pi_{2} \alpha(g)\right. \\
&\left.\cdot\left(x_{i+3}, x_{i+5}, \cdots\right), x_{i+4}, \cdots\right) \\
&=\left(x_{1}, \cdots, x_{i+1}, x_{i+2}, \pi_{1} \alpha(g)\left(x_{i+3}, x_{i+5}, \cdots\right), x_{i+4}, \pi_{2} \alpha(g)\right. \\
&\left.\cdot\left(x_{i+3}, x_{i+5}, \cdots\right), \cdots\right) .
\end{aligned}
$$

On the other hand, $f_{i+1}(x)=0$, so $\rho_{i+1}(x)=x$. Hence,

$$
\begin{aligned}
\rho_{i+1}^{-1} \sigma^{i+2}(\omega(\alpha(g))) \rho_{i+1}(x) & =\sigma^{i+2}(\omega(\alpha(g)))(x) \\
& =\left(x_{1}, x_{2}, \cdots, x_{i+2}, \pi_{1} \alpha(g)\left(x_{i+3}, x_{i+5}, \cdots\right),\right. \\
& \left.\quad x_{i+4}, \pi_{2} \alpha(g)\left(x_{i+3}, x_{i+5}, \cdots\right), \cdots\right) \\
& =\rho_{i}^{-1} \sigma^{i+1}(\omega(\alpha(g))) \rho_{i}(x),
\end{aligned}
$$

so the functions agree on the interfaces between the $K_{i}$, and $\beta$ is well defined. 
(b) $\beta$ is continuous on $G \times\left(X^{\infty}-Y\right)=\bigcup_{i \in N} G \times K_{i}$, because $\beta$ is continuous on each $G \times K_{i}$ and no three $K_{i}$ intersect. To see that $\beta$ is continuous on all of $G \times X^{\infty}$, there remains only to verify continuity on the closed set $G \times Y$. This is easy, because if $(g, y) \in G \times Y$ and if $U$ is a basic open set in $X^{\infty}$ containing $\beta(g, y)=y$, then there is an $n$ in $N$ such that $U=\tau_{n}^{-1}\left(\tau_{n}(U)\right)$, and if $V=U \cap U_{n}$, then $\beta: G \times V \rightarrow V \subset U$ because $V \subset\left(\cup_{i \geq n} K_{i}\right) \cup Y$ and $V=\tau_{n}^{-1}\left(\tau_{n}(V)\right)$ together imply that $\beta(G \times V)=V$.

(c) It is clear that $\beta$ inherits from $\alpha$ the property that for each $g$ and $h$ in $G, \beta(g h, x)=\beta(g, \beta(h, x))$ for each $x$ in $X^{\infty}$, and that $\beta$ defines a free action of $G$ on each $K_{i}$.

Combining (a), (b), and (c) yields that $\beta$ is a transformation group action of $G$ on $X^{\infty}$ which is semifree with fixed-point set $Y$.

Corollary 2.1. If $G$ is a topological group, $X$ is a space with the Reflective Isotopy Property, and $Z$ is a space such that $Z \times X^{n}$ is perfectly normal for each $n$ in $N$, then each closed subset of $Z \times X^{\infty}$ is the fixed-point set of a semifree transformation group action of $G$ if there is a free action of $G$ on $X^{\infty}$.

Corollary 2.2. If $G, X$ and $Z$ are as in Corollary 2.1, then each closed subset of $Z \times X^{\infty}$ is the union of the degenerate (single-point) orbits of an action of $G$ if there is an action of $G$ on $X^{\infty}$ without degenerate orbits.

Corollary 2.3. If $X$ is a metrizable, linear, topological space and if $G$ is a topological group, then each closed subset of $X^{\infty}$ is the fixed-point set of a semifree action of $G$ if there is a free action of $G$ on $X^{\infty}$ and is the union of the degenerate orbits of an action of $G$ if there is an action of $G$ on $X^{\infty}$ without degenerate orbits.

Proof. Every linear topological space $Y$ has the Reflective Isotopy Property. This may be seen as follows: Let $T^{\prime}$ be the homeomorphism on $Y^{\infty}$ defined by the formula $T^{\prime}\left(y_{1}, y_{2}, \cdots\right)=\left(y_{2},-y_{1}, y_{4},-y_{3}, \cdots\right)$; $T^{\prime}$ is invertibly isotopic to the identity by the function $H: Y^{\infty} \times I \rightarrow Y^{\infty}$ such that for each $\left(y_{1}, \cdots\right)$ in $Y^{\infty}$ and $t$ in $I$,

$$
\begin{aligned}
H\left(\left(y_{1}, \cdots\right), t\right)=( & \cos (t \pi / 2) y_{1}+\sin (t \pi / 2) y_{2}, \\
& -\sin (t \pi / 2) y_{1}+\cos (t \pi / 2) y_{2}, \cos (t \pi / 2) y_{3} \\
& \left.+\sin (t \pi / 2) y_{4},-\sin (t \pi / 2) y_{3}+\cos (t \pi / 2) y_{4}, \cdots\right) .
\end{aligned}
$$

The function $H$ acts independently on $Y_{2 n-1} \times Y_{2 n} \times I$ for each $n \geqq 1$, and on $Y_{2 n-1} \times Y_{2 n} \times I$, the structure of $Y$ as a linear topological space ensures continuity. Therefore, $H$ is continuous on $Y^{\infty} \times I$. Similarly, the function $G: Y^{\infty} \times I \rightarrow Y^{\infty}$ defined by 
$G\left(\left(y_{1}, \cdots\right), t\right)=\left(\cos (t \pi / 2) y_{1}-\sin (t \pi / 2) y_{2}\right.$,

$$
\begin{array}{r}
\sin (t \pi / 2) y_{1}+\cos (t \pi / 2) y_{2}, \cos (t \pi / 2) y_{3}-\sin (t \pi / 2) y_{4}, \\
\left.\sin (t \pi / 2) y_{3}+\cos (t \pi / 2) y_{4}, \cdots\right)
\end{array}
$$

is continuous. As $H\left(G\left(\left(y_{1}, \cdots\right), t\right), t\right)=G\left(H\left(\left(y_{1}, \cdots\right), t\right), t\right)$ $=\left(y_{1}, \cdots\right)$ for each $\left(y_{1}, \cdots\right)$ in $Y^{\infty}$ and $t$ in $I, H$ is an invertible isotopy on $Y^{\infty}$, and since $T=T^{\prime} \omega\left(\left(T^{\prime}\right)^{2}\right), T$ is also invertibly isotopic to the identity. Thus $Y$ has the Reflective Isotopy Property. (This is essentially what Wong did [10] to show that the real line has his version of the Reflective Isotopy Property.)

The space $X$ is a metrizable linear topological space and so meets the hypotheses of Theorem 2.1; Corollary 2.3 follows.

3. A theorem for separable, infinite-dimensional, Frechet spaces. A Frechet space is a completely metrizable, locally convex, linear topological space. It has recently been shown ([1] or [3], [5] or [6], and [7]) that all separable, infinite-dimensional Frechet spaces are homeomorphic to $R^{\infty}$, where $R$ is the real line. In this section, it is shown that if $G$ is a topological group and there is an effective transformation group action of $G$ on $R^{\infty}$, then there is a free action of $G$ on $R^{\infty}$. Combining this with Corollary 2.3 and the above-mentioned results yields the theorem touted in the introduction.

It will be notationally convenient to consider the space $\left(R^{\infty}\right)^{\infty}$ even though it is easily seen to be homeomorphic to $R^{\infty}$, so to simplify notation and for clarity let $s=R^{\infty}$.

THEOREM 3.1. If $X$ is the subset of $s^{\infty}$ composed of all points whose set of coordinates is dense in $s$, then $X$ is homeomorphic to $s$.

Proof. Let $\left\{y_{i}\right\}_{i \in N}$ be a countable, dense set in $s$, and for each $i$, let $\left\{U_{i j}\right\}_{j \in N}$ be a neighborhood base for $y_{i}$ composed of basic open sets of $s$, that is, each $U_{i j}$ is to be $\prod_{k \in N} \pi_{k}\left(U_{i j}\right)$, and for all but finitely many $k$ 's, $\pi_{k}\left(U_{i j}\right)=R_{k}$. Let $Y_{i j}=\left\{y \in s^{\infty} \mid \pi_{k}(y) \notin U_{i j}\right.$ for each $k$ in $\left.N\right\}$. Each $Y_{i j}$ is closed in $s^{\infty}$, and $\bigcup_{i, j \in N} Y_{i j}=s^{\infty}-X$. Let $\phi: N \rightarrow N \times N$ be a function such that each point inverse of $\phi$ is infinite, and for each $k \in N$, let $\psi_{k}$ be a homeomorphism of $s_{k}$ onto itself which carries $s_{k}-U_{\phi(k)}$ into a set which is bounded in some coordinate of $s_{k}$. This may be done easily because $U_{\phi(k)}$ is a basic open set in $s_{k}$. Now, let $\psi$ be the homeomorphism of $s^{\infty}$ onto itself which is the product of the $\psi_{k}$, that is, $\psi\left(y_{1}, y_{2}, \cdots\right)=\left(\psi_{1}\left(y_{1}\right), \psi_{2}\left(y_{2}\right), \cdots\right)$ for each point $\left(y_{1}, y_{2}, \cdots\right)$ in $s^{\infty}$. Because $s^{\infty}$ may be regarded as a "refactorization" of $R^{\infty}$ induced by a set isomorphism between $N$ and $N \times N$, the 
homeomorphism $\psi$ on $s^{\infty}$ may be regarded as being or inducing one on $R^{\infty}$ which has the property that the image of each $Y_{i j}$ is a set which is bounded in infinitely many coordinates (in $R^{\infty}$ ). A theorem of R. D. Anderson [2] asserts that in $R^{\infty}$ the complement of a countable union of such closed sets is homeomorphic to $R^{\infty}$. In this case, the complement is the homeomorphic image of $X$, which is therefore homeomorphic to $s$.

ThEOREM 3.2. If $X$ is a separable, infinite-dimensional, Frechet space and if $G$ is a topological group for which there is an effective transformation group action on $X$, then each closed subset of $X$ is the fixed-point set of a semifree action of $G$ on $X$.

Proof. As remarked above, because $X$ is homeomorphic to $R^{\infty}$, Theorem 2.1 reduces the problem to that of showing that if $G$ is a topological group for which there is an effective action on $R^{\infty}$, then there is a free action of $G$ on $R^{\infty}$. Therefore, let $\alpha$ be an effective transformation group action of the topological group $G$ on $R^{\infty}=s$. Let $\beta$ be the product action of $G^{\infty}$ on $s^{\infty}$ induced from $\alpha$, that is, if $\left(g_{1}, \cdots\right)$ is in $G^{\infty}$ and $\left(y_{1}, \cdots\right)$ is in $s^{\infty}$,

$$
\beta\left(\left(g_{1}, \cdots\right),\left(y_{1}, \cdots\right)\right)=\left(\alpha\left(g_{1}, y_{1}\right), \alpha\left(g_{2}, y_{2}\right), \cdots\right) .
$$

Let $\gamma: G \rightarrow G^{\infty}$ be the diagonal embedding of $G$ in $G^{\infty}$, and let $\delta$ be the action of $G$ on $s^{\infty}$ defined by first sending $G$ into $G^{\infty}$ with $\gamma$ and then applying $\beta$. Let $Y$ be the subset of $s^{\infty}$ composed of all points whose set of coordinates is dense in $s$. By Theorem 3.1, $Y$ is homeomorphic to $s=R^{\infty}$ (and hence to $X$ ). Furthermore, $\delta:\{g\} \times Y \rightarrow Y$ for all $g$ in $G$, and because $\alpha$ is effective, $\delta \mid G \times Y$ is a free action of $G$ on $Y$ as a transformation group. This proves the theorem.

COROLlaRY 3.1. If $G$ is any compact metric group and $Y$ is any closed subset of the separable, infinite-dimensional, Frechet space $X$, there is a semifree action of $G$ on $X$ as a transformation group with fixed-point set $Y$.

Proof. Let $G L_{n}$ be the group of all linear homeomorphisms of $R^{n}$ onto itself under the topology of uniform convergence on bounded closed sets, and let $C$ be the group $\prod_{n \in N}\left(G L_{n}\right)^{\infty}$. This group acts effectively on $\prod_{n \in N}\left(R^{n}\right)^{\infty}$ by the product of the individual actions. The latter space, however, is homeomorphic to $R^{\infty}$. Also, it is well known (see, for example, $[9$, p. 100]) that any compact, metric group can be isomorphically embedded in $C$. Using this, and the fact that all separable, infinite-dimensional, Frechet spaces are homeomorphic, Theorem 3.2 applies to give the desired result. 


\section{REFERENCES}

1. R. D. Anderson, Hilbert space is homeomorphic to the countable infinite product of lines, Bull. Amer. Math. Soc. 72 (1966), 515-519.

2. - Topological properties of the Hilbert cube and the infinite product of open intervals, Trans. Amer. Math. Soc. 126 (1967), 200-216.

3. R. D. Anderson and R. H. Bing, A complete, elementary proof that Hilbert space is homeomorphic to the countable infinite product of lines, Bull. Amer. Math. Soc. 74 (1968), 771-792.

4. A. Beck, On invariant sets, Ann. of Math. 67 (1958), 99-103.

5. C. Bessaga, On topological classification of complete linear metric spaces, Fund. Math. 56 (1965), 251-288.

6. C. Bessaga and A. Pełczynski, Some remarks on homeomorphism of F-spaces, Bull. Acad. Polon. Sci. Ser. Sci. Math. Astronom. Phys. 10 (1962), 265-270.

7. M. I. Kadec, On topological equivalence of all separable Banach spaces, Dokl. Akad. Nauk SSSR 167 (1966), 23-25=Soviet Math. Dokl. 7 (1966), 319-322.

8. V. L. Klee, Jr., Fixed point sets of periodic homeomorphisms of Hilbert space, Ann. of Math. (2) 64 (1956), 393-395.

9. D. Montgomery and L. Zippin, Topological transformation groups, Interscience, New York, 1955.

10. R. Y.-T. Wong, On homeomorphisms of infinite dimensional spaces, Trans. Amer. Math. Soc. 128 (1967), 148-154.

Louisiana State University 